\title{
Location and Layout of Common Storage and Multichannel Common Distribution considering Time Windows
}

\author{
Biqin Hu $\mathbb{D}$, ${ }^{1}$ Bin Yang $\mathbb{D}^{2}$, Wei Jiang $\mathbb{D}^{3}$ Zhe Yang $\mathbb{D}^{4},{ }^{4}$ and Mohammed Abdella Kemal $\mathbb{D}^{5}$ \\ ${ }^{1}$ Institute of Logistics Science and Engineering, Shanghai Maritime University, Shanghai Pudong, 201306, China \\ ${ }^{2}$ Business School, Zhejiang Textile and Fashion College, Ningbo 315211, China \\ ${ }^{3}$ CIM Department, Shanghai Advanced Semiconductor Manufacturing Co., Ltd., Shanghai Pudong, 201206, China \\ ${ }^{4}$ School of Computer Science, University of Manchester, Oxford Road, Manchester, M13 9PL, UK \\ ${ }^{5}$ Arba Minch University, Ethiopia \\ Correspondence should be addressed to Mohammed Abdella Kemal; abdella.kemal@amu.edu.et
}

Received 10 May 2021; Accepted 15 June 2021; Published 6 July 2021

Academic Editor: Vimal Shanmuganathan

Copyright (C) 2021 Biqin Hu et al. This is an open access article distributed under the Creative Commons Attribution License, which permits unrestricted use, distribution, and reproduction in any medium, provided the original work is properly cited.

\begin{abstract}
In the process of logistic activities of common storage and common distribution, due to problems such as urban traffic congestion period, parking restriction, working time restriction, and road restriction time, people often agree on the arrival time for customer orders, so that the receiving and delivering parties can connect in time and speed up unloading and distribution. Therefore, considering the actual distribution operation, it is necessary to consider the location and layout of common storage and multichannel common distribution in time windows. This paper takes multichannel common storage and common distribution as the research object, combines with the actual distribution situation, considers the location and distribution route optimization of the common storage and common distribution mode under the condition of time windows, takes the form of linking time windows with distribution time completion efficiency, and forms a reverse constraint on the objective function by time completion efficiency, thus realizing the double-objective optimization of "minimizing the total cost and satisfying the time windows." In addition, through the comparison of various intelligent algorithms, find that the squirrel search algorithm (SSA) has more stable performance and better solution results.
\end{abstract}

\section{Introduction}

With the management and control of distribution vehicles in large cities becoming more and more strict, urban distribution vehicles are affected by high parking and loading charges, the shortage of vehicle entrance passes indexes and the like; meanwhile, a large number of cross distribution and personnel overlap among logistic enterprises cause the unnecessary urban end distribution cost, and the demand of end distribution among the logistic distribution enterprises is generated (see, e.g., [1]). Secondly, under the new retail trend, higher requirements are put forward for the timeliness of the logistic distribution, and the service requirements of hourly arrival, daily arrival, and next day arrival are common, which makes the new retail mode integrating online and offline urgently need an efficient common storage and distribution system as a support (see, e.g., [2]). Under this background, the channel boundaries of the urban logistic distribution gradually show a trend of blurring and integration, and common storage effectively acts as the function of multichannel cargoes collection and distribution in the process of channel integration (see, e.g., [3]). Through common storage, joint holding of inventory and joint use of storage facilities and management personnel, inventory costs, and labor costs can be effectively reduced, and order response speed can be improved. At the same time, combined with the common distribution, the transportation distribution plan is formulated from the perspective of storage and distribution integration operation optimization, and the same destination-oriented cargoes are distributed with one vehicle, which not only reduces repeated distribution but also greatly saves limited transport resources (see, e.g., [4]).

In the literature of integrated optimization of storage and distribution, the storage location problem is a mixed 
for $\mathrm{i}=1$ :length (Ware House) no_WH=Ware House(i);

no_WH_N=find (WH_N==i); [ ,temp_p] $=\operatorname{sort}(\mathrm{x}($ no_WH_N,3));

no_WH_N=no_WH_N (temp_p); num_V=data.WareHouse (no_WH,4);

recording.V $\{\mathrm{i}\}=$ zeros (num_V,10); S_WH=data.WareHouse $($ no_WH,2);

for $j=1$ :length (no_WH_N).

if no_WH_N $(j)<=$ data.num_WH continue;

end no_N=no_WH_N(j);

Type_Cargoes=data.Demand (no_N,5); weight_Cargoes=.

(data.Demand(no_N,2:3)); [ ,priority_V]=sor $\mathrm{t}(\mathrm{x}($ no_N $, 4: 3+$ num_V $)$ );

Algorithm 1

$\mathrm{x}=$ cell(option.popSize,1); fit=zeros(option.popSize,1); for $\mathrm{j}=1$ :option.popSize .

$x\{j, 1\}=$ creat_x_1(option,data); $v\{j, 1\}=\operatorname{rand}(\operatorname{size}(x\{j, 1\}))$;

fit $(j, 1)=$ option.aimFcn $(x\{j, 1\}$,option,data $)$;

end data. $\mathrm{x}=\mathrm{x}$; data.fit $=$ fit; data. $\mathrm{v}=\mathrm{v}$;

Algorithm 2

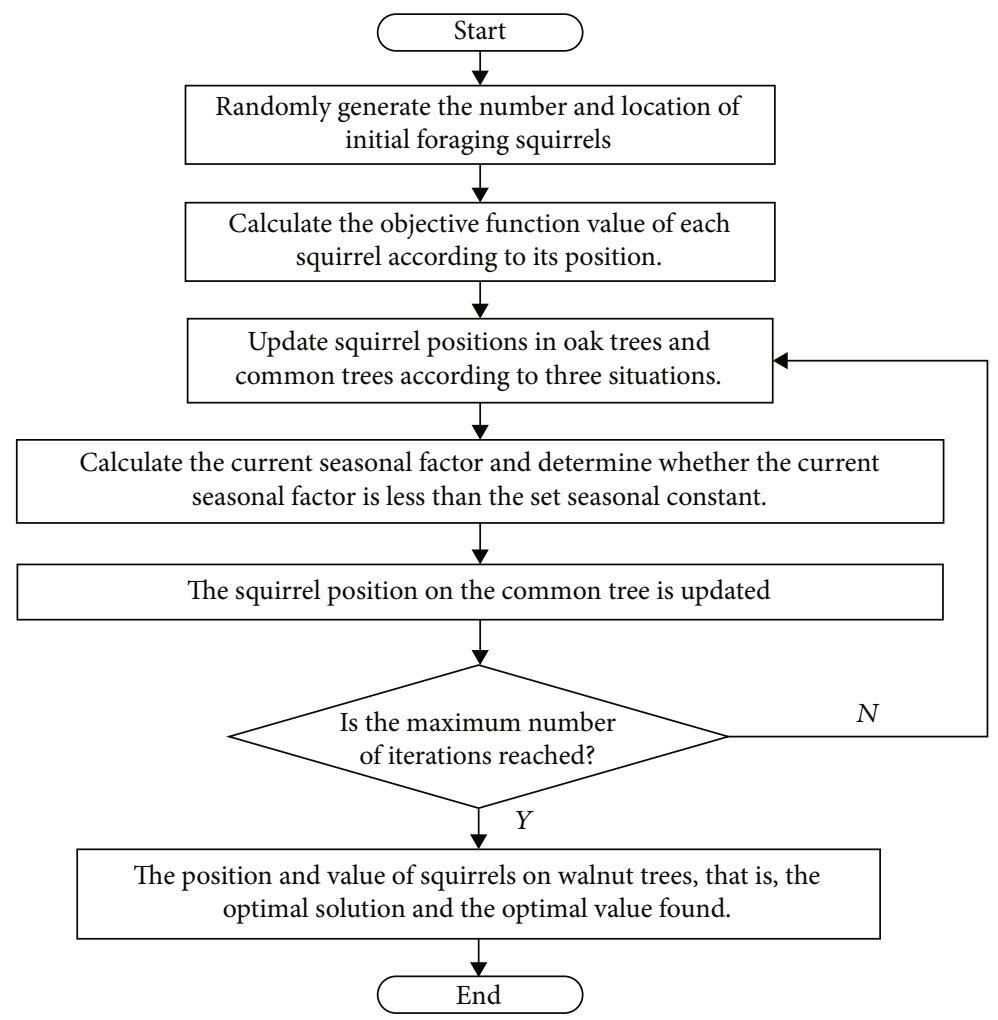

FIGURE 1: Solving steps of the squirrel search algorithm for the multichannel common storage and subchannel distribution with location and layout problem.

integer programming problem, while the distribution routing problem is a vehicle routing problem (VRP); so, it is a NP problem to combine the two problems. The traditional problem of warehouse location and distribution is generally solved by disassembly into two stages or consider the influence of production place, inventory level, and distribution channel when selecting warehouse location, but most of them are not optimized together (see e.g. [5]). Most of the warehouse location models currently established are based on the freight or service level under single channel circulation mode, proposing the selection of warehouse location, but less research has been carried out from the perspective of storage and distribution integration. 
TABLE 1: Time window plan for each demand point.

\begin{tabular}{|c|c|c|c|c|c|c|c|}
\hline Demand point & $P=1$, pallet & $P=2$, items $/$ parcels & $\begin{array}{c}\text { Estimated } \\
\text { unloading time }\end{array}$ & $\begin{array}{l}\text { Hard time } \\
\text { window } 1\end{array}$ & $\begin{array}{l}\text { Soft time } \\
\text { window } 1\end{array}$ & $\begin{array}{l}\text { Soft time } \\
\text { window } 2\end{array}$ & $\begin{array}{l}\text { Soft time } \\
\text { window } 3\end{array}$ \\
\hline 11 & 31 & 0 & 1 & 0.5 & 1 & 6 & 16 \\
\hline 12 & 31 & 0 & 1 & 0.5 & 1 & 6 & 16 \\
\hline 13 & 28 & 0 & 1 & 0.5 & 1 & 6 & 16 \\
\hline 14 & 33 & 0 & 1 & 0.5 & 1 & 6 & 16 \\
\hline 15 & 14 & 0 & 1 & 0.5 & 1 & 6 & 16 \\
\hline 16 & 10 & 0 & 1 & 0.5 & 1 & 6 & 16 \\
\hline 17 & 2 & 0 & 1 & 0.5 & 1 & 6 & 16 \\
\hline 18 & 2 & 0 & 1 & 0.5 & 1 & 6 & 16 \\
\hline 19 & 12 & 0 & 1 & 0.5 & 1 & 6 & 16 \\
\hline 20 & 30 & 0 & 1 & 0.5 & 1 & 6 & 16 \\
\hline 21 & 3 & 0 & 1 & 0.5 & 1 & 6 & 16 \\
\hline 22 & 3 & 0 & 1 & 0.5 & 1 & 6 & 16 \\
\hline 23 & 4 & 0 & 1 & 0.5 & 1 & 6 & 16 \\
\hline 24 & 31 & 0 & 1 & 0.5 & 1 & 6 & 16 \\
\hline 25 & 40 & 0 & 1 & 0.5 & 1 & 6 & 16 \\
\hline 26 & 0 & 19 & 1 & 0.5 & 1 & 6 & 16 \\
\hline 27 & 0 & 11 & 1 & 0.5 & 1 & 6 & 16 \\
\hline 28 & 0 & 18 & 1 & 0.5 & 1 & 6 & 16 \\
\hline 29 & 0 & 2 & 1 & 0.5 & 1 & 6 & 16 \\
\hline 30 & 0 & 2 & 1 & 0.5 & 1 & 6 & 16 \\
\hline 31 & 0 & 32 & 1 & 0.5 & 1 & 6 & 16 \\
\hline 32 & 0 & 3 & 1 & 0.5 & 1 & 6 & 16 \\
\hline 33 & 0 & 40 & 1 & 0.5 & 1 & 6 & 16 \\
\hline 34 & 0 & 15 & 1 & 0.5 & 1 & 6 & 16 \\
\hline 35 & 0 & 4 & 1 & 0.5 & 1 & 6 & 16 \\
\hline 36 & 0 & 6 & 1 & 0.5 & 1 & 6 & 16 \\
\hline 37 & 0 & 28 & 1 & 0.5 & 1 & 6 & 16 \\
\hline 38 & 0 & 3 & 1 & 0.5 & 1 & 6 & 16 \\
\hline 39 & 0 & 24 & 1 & 0.5 & 1 & 6 & 16 \\
\hline 40 & 0 & 29 & 1 & 0.5 & 1 & 6 & 16 \\
\hline 41 & 5 & 0 & 1 & 0.5 & 1 & 6 & 16 \\
\hline 42 & 3 & 0 & 1 & 0.5 & 1 & 6 & 16 \\
\hline 43 & 2 & 0 & 1 & 0.5 & 1 & 6 & 16 \\
\hline
\end{tabular}

Multichannel means that the structure and mode of circulation are different. For example, the logistic demand of online channels is small-piece high-frequency mode, while the logistic demand of offline stores is large-piece lowfrequency mode. The joint storage of online channels and offline channels can reduce the inventory of the same product and realize the joint storage and distribution of the two modes (see, e.g., [6]). However, this requires redesigning the distribution network and reasonably selecting the warehouse location and optimizing the distribution route according to the characteristics of multichannel integrated warehouse allocation. At present, there are few literatures on the research content of the integrated layout of common storage and distribution under the background of multichannel integration (see, e.g., [7]). Most of them expound the characteristics and development trend of multichannel
TABLE 2: Available area, rental, and vehicles of warehouse candidate points.

\begin{tabular}{lccc}
\hline Warehouse No. & Area & Area rent & Number of vehicles \\
\hline 1 & 1000 & 2.5 & 5 \\
2 & 1500 & 1.3 & 5 \\
3 & 1600 & 0.8 & 5 \\
4 & 1100 & 0.7 & 5 \\
5 & 800 & 1.2 & 5 \\
6 & 2000 & 1.1 & 5 \\
7 & 1200 & 1.3 & 5 \\
8 & 2800 & 1.0 & 5 \\
9 & 3000 & 1.5 & 5 \\
10 & 3200 & 0.6 & 5 \\
\hline
\end{tabular}


TABLE 3: Results of distribution cargo types and transportation distance of vehicles corresponding to candidate warehouse No. 3.

\begin{tabular}{lcccc}
\hline Vehicle & Number of access nodes & Quantity of type 1 cargoes & Quantity of type 2 cargoes & Single distribution distance \\
\hline 1 & 4 & 57 & 0 & 44.51 \\
2 & 4 & 35 & 34 & 73.39 \\
3 & 4 & 101 & 0 & 74.12 \\
4 & 2 & 3 & 3 & 54.14 \\
\hline
\end{tabular}

TABLE 4: Distribution route points of vehicles corresponding to candidate warehouse No. 3.

\begin{tabular}{lccccc}
\hline Vehicle & Route point 1 & Route point 2 & Route point 3 & Route point 4 & Route point 5 \\
\hline 1 & 12 & 18 & 15 & 16 & 14 \\
2 & 43 & 37 & 36 & 11 & 13 \\
3 & 19 & 0 & 0 & 0 \\
4 & 38 & 42 & 0 & 0 \\
\hline
\end{tabular}

TABle 5: Results of distribution cargo types and transportation distance of vehicles corresponding to candidate warehouse No. 10.

\begin{tabular}{lcccc}
\hline Vehicle & Number of access nodes & Quantity of type 1 cargoes & Quantity of type 2 cargoes & Single distribution distance \\
\hline 1 & 4 & 35 & 6 & 55.83 \\
2 & 5 & 0 & 96 & 37.55 \\
3 & 5 & 8 & 40 & 30.26 \\
4 & 5 & 45 & 57 & 36.78 \\
\hline
\end{tabular}

storage and distribution services, but there are few researches on the cost of the multichannel logistic distribution and single-channel logistic distribution. Some scholars discussed the service mode of multichannel orders and store-based or warehouse-based distribution systems. Under the condition of limited space, professional order pickers and ordinary customers will influence each other in the store-based model; so, it is necessary to seek cooperative work strategies (see, e.g., [8]). Alptekinoglu and Tang (2005) proposed a model to analyze the multichannel distribution system and used the joint distribution system of two retailers to find a low-cost product distribution method. Relevant literatures show that there are many methods to solve the storage location or distribution routing problem. However, for the integrated optimization of storage and distribution, there is almost no very effective approximate optimal algorithm in a short period of time. squirrel search algorithm is a new algorithm inspired by squirrel's dynamic foraging behavior. Compared with existing algorithms, this algorithm has more accurate solution and fast convergence speed in big data experiments, but it has not yet been applied to storage and distribution optimization problems (see, e.g., [9]).

For the optimization of location and distribution route in the mode of common storage and common distribution under the condition of time windows, it is not only necessary to consider the reasonable location of warehouses under the condition of multichannel logistic demand, but also to consider the layout of the corresponding multichannel distribution network, and to study the cooperative operation and common optimization among warehouses, vehicles, and demand points. In the existing subchannel distribution pro- cess, we need to combine the service time requirements, consider the optimization and operation of storage and distribution integration resources under the condition of time windows on the basis of common storage and common distribution, and study the warehouse location, distribution network layout, distribution plan formulation, and other contents of the multichannel common storage and distribution under the condition of time constraint (see, e.g., [10]).

On the basis of a common storage multichannel common distribution location and layout model, a demand point receiving time window is added to the problem, the earliest service time, the latest service time and the running time of a vehicle of each demand point are combined, the minimum total cost of distribution and storage is taken as a target, whether the arrival time of the vehicle is linked with the completion efficiency of the distribution time within the appointed receiving time is set as the reverse relation of a target function, the distribution route is optimized, the distribution vehicle resources are reasonably distributed, and the arrival time and the departure time of the vehicle to the demand point are as much as possible in accordance with the time interval of allowable cargoes receiving of each demand point, thus achieving the dual objectives of meeting the time window and the lowest cost.

\section{Material and Methods}

2.1. Objective Function. The objective function consists of the total cost of warehouse allocation and the time completion efficiency, and the total cost is divided into two parts. The first part is the distribution cost. The transportation distance 
TABLE 6: Distribution route points of vehicles corresponding to candidate warehouse No. 10.

\begin{tabular}{|c|c|c|c|c|c|}
\hline Vehicle & Route point 1 & Route point 2 & Route point 3 & Route point 4 & Route point 5 \\
\hline 1 & 35 & 23 & 24 & 30 & 0 \\
\hline 2 & 29 & 26 & 31 & 32 & 33 \\
\hline 3 & 27 & 21 & 22 & 40 & 17 \\
\hline 4 & 39 & 41 & 34 & 25 & 28 \\
\hline
\end{tabular}

TABle 7: Distribution time and time completion efficiency of each demand point.

\begin{tabular}{|c|c|c|c|c|c|c|c|}
\hline Demand point & $\begin{array}{c}\text { Distribution } \\
\text { completion time }\end{array}$ & $\begin{array}{c}\text { Time completion } \\
\text { efficiency }\end{array}$ & $\begin{array}{c}\text { Estimated } \\
\text { unloading time }\end{array}$ & $\begin{array}{l}\text { Hard time } \\
\text { window } 1\end{array}$ & $\begin{array}{l}\text { Soft time } \\
\text { window } 1\end{array}$ & $\begin{array}{l}\text { Soft time } \\
\text { window } 2\end{array}$ & $\begin{array}{l}\text { Soft time } \\
\text { window } 3\end{array}$ \\
\hline 11 & 4.30 & 1.00 & 1 & 0.5 & 1 & 6 & 16 \\
\hline 12 & 1.53 & 1.00 & 1 & 0.5 & 1 & 6 & 16 \\
\hline 13 & 5.36 & 1.00 & 1 & 0.5 & 1 & 6 & 16 \\
\hline 14 & 5.33 & 1.00 & 1 & 0.5 & 1 & 6 & 16 \\
\hline 15 & 3.58 & 1.00 & 1 & 0.5 & 1 & 6 & 16 \\
\hline 16 & 4.58 & 1.00 & 1 & 0.5 & 1 & 6 & 16 \\
\hline 17 & 5.63 & 1.00 & 1 & 0.5 & 1 & 6 & 16 \\
\hline 18 & 2.56 & 1.00 & 1 & 0.5 & 1 & 6 & 16 \\
\hline 19 & 3.14 & 1.00 & 1 & 0.5 & 1 & 6 & 16 \\
\hline 20 & 1.90 & 1.00 & 1 & 0.5 & 1 & 6 & 16 \\
\hline 21 & 2.26 & 1.00 & 1 & 0.5 & 1 & 6 & 16 \\
\hline 22 & 3.33 & 1.00 & 1 & 0.5 & 1 & 6 & 16 \\
\hline 23 & 2.40 & 1.00 & 1 & 0.5 & 1 & 6 & 16 \\
\hline 24 & 3.44 & 1.00 & 1 & 0.5 & 1 & 6 & 16 \\
\hline 25 & 4.60 & 1.00 & 1 & 0.5 & 1 & 6 & 16 \\
\hline 26 & 2.34 & 1.00 & 1 & 0.5 & 1 & 6 & 16 \\
\hline 27 & 1.15 & 1.00 & 1 & 0.5 & 1 & 6 & 16 \\
\hline 28 & 5.75 & 1.00 & 1 & 0.5 & 1 & 6 & 16 \\
\hline 29 & 1.15 & 1.00 & 1 & 0.5 & 1 & 6 & 16 \\
\hline 30 & 4.90 & 1.00 & 1 & 0.5 & 1 & 6 & 16 \\
\hline 31 & 3.49 & 1.00 & 1 & 0.5 & 1 & 6 & 16 \\
\hline 32 & 4.51 & 1.00 & 1 & 0.5 & 1 & 6 & 16 \\
\hline 33 & 5.56 & 1.00 & 1 & 0.5 & 1 & 6 & 16 \\
\hline 34 & 3.37 & 1.00 & 1 & 0.5 & 1 & 6 & 16 \\
\hline 35 & 1.31 & 1.00 & 1 & 0.5 & 1 & 6 & 16 \\
\hline 36 & 3.70 & 1.00 & 1 & 0.5 & 1 & 6 & 16 \\
\hline 37 & 2.63 & 1.00 & 1 & 0.5 & 1 & 6 & 16 \\
\hline 38 & 1.68 & 1.00 & 1 & 0.5 & 1 & 6 & 16 \\
\hline 39 & 1.23 & 1.00 & 1 & 0.5 & 1 & 6 & 16 \\
\hline 40 & 4.48 & 1.00 & 1 & 0.5 & 1 & 6 & 16 \\
\hline 41 & 2.28 & 1.00 & 1 & 0.5 & 1 & 6 & 16 \\
\hline 42 & 2.81 & 1.00 & 1 & 0.5 & 1 & 6 & 16 \\
\hline 43 & 1.23 & 1.00 & 1 & 0.5 & 1 & 6 & 16 \\
\hline
\end{tabular}

between all points on the route is multiplied by the unit distance transportation cost of the vehicles carried. The second part is the storage cost, the total rental cost of the warehouse candidate point $i$ multiplied by whether to locate the site as the decision variable of the warehouse (see, e.g., [11]). Time completion efficiency is the average time completion efficiency of distribution points. The higher the time completion efficiency, the smaller the objective function value, which can effectively achieve the dual requirements of minimum cost and meeting the distribution time window. 


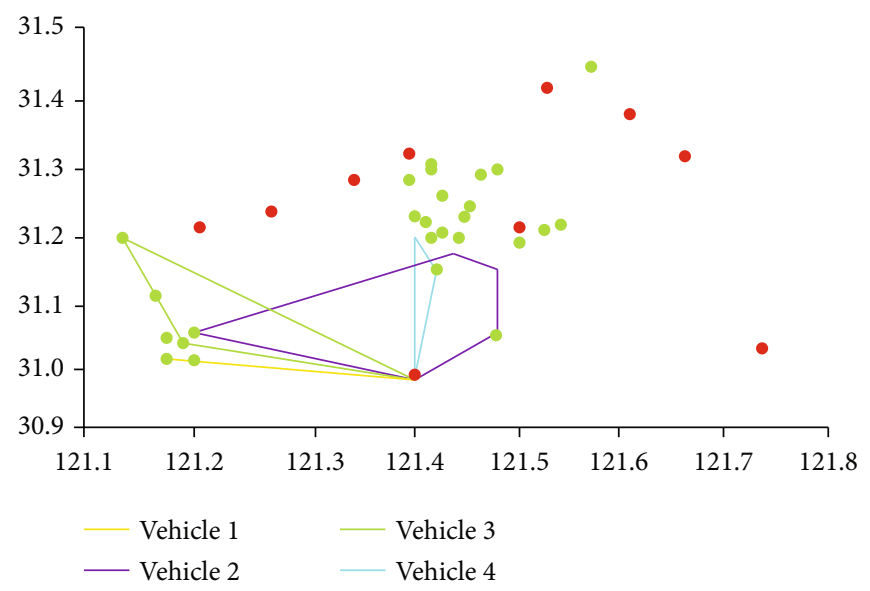

FIGURE 2: Distribution route diagram corresponding to candidate warehouse No. 3.

2.2. Description of Time Window Restriction Conditions. The restriction condition 1 refers to if the distribution vehicle corresponds to the service demand point, and then the completion time point of the distribution vehicle to the demand point is equal to the completion time point of the distribution vehicle to the demand point plus the service time of the distribution vehicle at the demand point and the travel time of the distribution vehicle to travel from the demand point to the demand point (see, e.g., [12]).

The restriction condition 2 is a piecewise function for calculating the service time completion efficiency of the distribution point related to the soft and hard time windows. If the completion time of the vehicle at the demand point is less than the earliest time point (hard time window) or greater than the latest time point (maximum soft time window), the time completion efficiency is 0 . If the completion time of the vehicle at the demand point is greater than or equal to the earliest serviceable time point (hard time window) but less than the agreed start service time, the time completion efficiency is the deviation ratio of the current arrival time and the agreed start service time (see, e.g., [13]). If the completion time of the vehicle at the demand point is greater than the agreed end service time and less than the latest time point (maximum soft time window), the time completion efficiency is the deviation ratio between the completion time of the vehicle at the demand point and the agreed end service time. If the completion time of the vehicle at the demand point is between the agreed start service time and the agreed end service time, the time completion efficiency is 1 .

The restriction condition 3 means that the total distribution time completion efficiency is the average value of the time completion efficiency of each distribution point.

The restriction condition 4 means that if the distribution vehicle corresponds to the service demand point, the travel time from point to point on the path is equal to the distance between the two points divided by the average speed of the distribution vehicle.

\subsection{Squirrel Search Algorithm Solution Design}

2.3.1. Design Idea of the Squirrel Search Algorithm. The squirrel search algorithm assumes that there are squirrels on trees in the forest, and there is only one squirrel in each tree, and each squirrel forages for food and maximizes the available food resources through dynamic actions (see e.g. [14]). There are only three kinds of trees in the forest: Common tree, oak tree and walnut tree, of which oak tree and walnut tree are the food sources of squirrels, and only three oak trees and one walnut tree are set to exist in the forest area.

2.3.2. Solving Steps of the Squirrel Search Algorithm for the Multichannel Common Storage and Subchannel Distribution with Location and Layout Problem. Step 1. Randomly generate the position of squirrels. The position is represented by the dimensional independent variable matrix in the model, and the starting position is randomly generated as follows:

2.4. Design of Independent Variables. Randomly generates a matrix of independent variables $X$, for example, for 93 demand points and 10 warehouses, the generated $X$ matrix has $93+10=103$ rows and 8 columns, of which the first column value indicates the warehouse construction priority, the second column value indicates the corresponding relationship between the warehouse and the demand points, the third column value indicates the distribution route of the demand points, the fourth column value indicates that the demand points are responsible for distributing vehicles, and the fourth, fifth, sixth, seventh, and eighth column values indicate the sending sequence of vehicles.

2.5. Determine the Design of the Construction Warehouse. The $X$ values generated in the random first column of the warehouse are sorted. If the number with the value less than 0.5 is found, the candidate points in the top few places will be selected as the warehouse. For example, after warehouses 1-10 are sorted, there are 3 values less than 0.5 , and then the warehouse number corresponding to the 1-3 digits $x$ value of the sorted $B$.

Determine the corresponding relationship design between warehouse and demand point:

For the second column of the independent variable $X$ matrix, point multiplied by the number of built warehouses and rounded up. If the rounded value is greater than or equal 


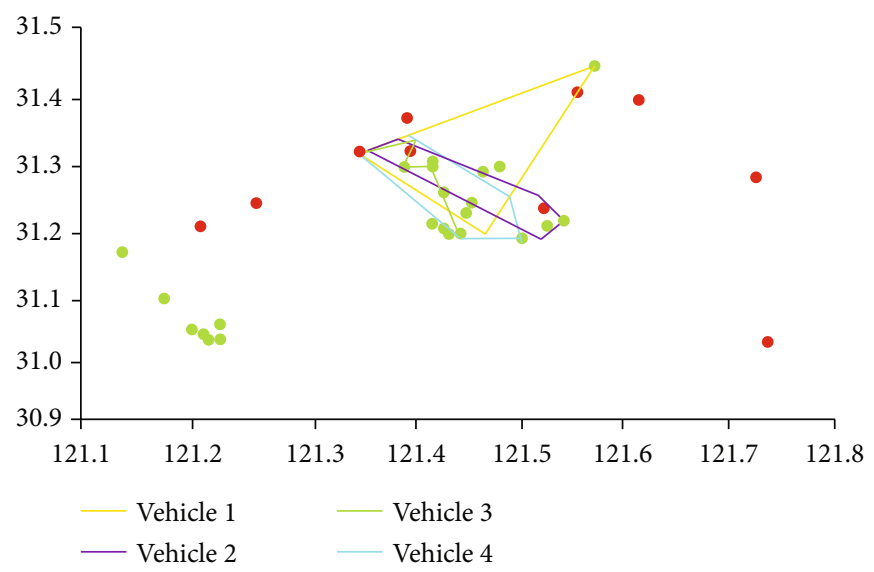

FIgURE 3: Distribution route diagram corresponding to candidate warehouse No. 10.

TABLE 8: Results of 5 algorithms under different data sizes.

\begin{tabular}{|c|c|c|c|c|c|c|}
\hline Size & Result & SSA & APSO & AGA & $\mathrm{CSO}$ & WPA \\
\hline \multirow{5}{*}{$10 \times 13$} & Best & 225.75 & 229.02 & 227.62 & 226.66 & 227.15 \\
\hline & Mean & 226.80 & 235.89 & 253.34 & 231.89 & 232.53 \\
\hline & Worst & 229.24 & 244.17 & 285.51 & 240.05 & 240.59 \\
\hline & Median & 226.62 & 237.39 & 260.09 & 231.84 & 231.86 \\
\hline & Time (10) & 997.40 & 276.54 & 269.80 & 275.03 & 2216.26 \\
\hline \multirow{5}{*}{$10 \times 33$} & Best & 1023.82 & 1045.34 & 1101.57 & 1045.78 & 1081.15 \\
\hline & Mean & 1067.59 & 1101.01 & 1175.62 & 1073.25 & 1129.51 \\
\hline & Worst & 1148.07 & 1152.34 & 1258.68 & 1130.05 & 1162.82 \\
\hline & Median & 1061.26 & 1106.71 & 1174.83 & 1072.36 & 1136.22 \\
\hline & Time(10) & 1111.50 & 301.15 & 335.55 & 359.67 & 2078.14 \\
\hline \multirow{5}{*}{$10 \times 53$} & Best & 2099.66 & 2211.38 & 2303.31 & 2096.97 & 2389.61 \\
\hline & Mean & 2191.58 & 2361.13 & 2427.04 & 2305.26 & 2461.44 \\
\hline & Worst & 2297.26 & 2648.55 & 2643.81 & 2669.60 & 2595.11 \\
\hline & Median & 2186.69 & 2342.83 & 2393.48 & 2265.50 & 2451.08 \\
\hline & Time (10) & 1098.11 & 351.56 & 358.77 & 366.86 & 2471.37 \\
\hline \multirow{5}{*}{$10 \times 73$} & Best & 3270.64 & 3611.38 & 3531.92 & 3547.04 & 3687.64 \\
\hline & Mean & 3485.94 & 3786.80 & 3655.94 & 3863.75 & 3893.35 \\
\hline & Worst & 3597.42 & 4103.18 & 3918.77 & 4165.65 & 4128.85 \\
\hline & Median & 3501.19 & 3768.78 & 3604.21 & 3852.54 & 3916.60 \\
\hline & Time (10) & 2866.76 & 973.84 & 972.20 & 968.80 & 6365.26 \\
\hline \multirow{5}{*}{$10 \times 93$} & Best & 4789.20 & 5132.77 & 5311.89 & 5196.20 & 5618.54 \\
\hline & Mean & 5160.04 & 5404.58 & 5648.84 & 5615.16 & 5847.26 \\
\hline & Worst & 5461.17 & 5821.07 & 5910.29 & 5842.58 & 6128.73 \\
\hline & Median & 5147.29 & 5320.58 & 5635.41 & 5632.35 & 5817.87 \\
\hline & Time (10) & 3326.43 & 1116.46 & 1109.59 & 1105.34 & 7066.79 \\
\hline
\end{tabular}

to the number of built warehouses, it means that the built warehouse is responsible for this demand point.

Design of distribution route, number of vehicles, and priority of vehicle distribution for each demand point are as follows:

Sort the values in the third column of the independent variable $X$ matrix, obtain the order in which the points are visited according to the sorting results, and eliminate the warehouse candidate points (see, e.g., [15]). And we give feedback to the 4 th column value of the independent variable $X$ matrix as the number of vehicles to be delivered and sort the column values from the 4 th column to the " $3+$ number of vehicles" to indicate the priority of vehicles to be delivered. 
Initial position call, value, and acceleration generation design of squirrel are as follows:

The initial population has been generated. The step of the squirrel search algorithm is shown in Figure 1.

\section{Results}

3.1. Experimental Data. The cargoes demand information, unloading time, the earliest service time point (hard time window 1), and the agreed service time point, i.e., soft time windows 1 and 2 and the latest reception time (soft time window) of each demand point are shown in Table 1 . Table 2 shows the available area, current, and vehicles of warehouse candidate points.

3.2. Example Results. By using the solution steps of the squirrel search algorithm in the fifth part, the examples of the above 10 warehouse candidate points and 33 demand points are solved. According to 10 experiments, the best solution is obtained, and the No. 3 candidate point and No. 10 candidate point are selected to set up the warehouse, as shown in the following Tables 3-7. Table 3 shows the type of cargoes, number of access nodes, number of cargoes carried, and single distribution distance corresponding to candidate warehouse 3 . Table 4 shows the vehicle distribution route corresponding to candidate warehouse 3 . The specific route and longitude and latitude of route points are shown in Figure 2, and the warehouse 3 is equipped with 4 vehicles. Table 5 shows the type of cargoes, number of access nodes, number of cargoes carried, and single distribution distance corresponding to candidate warehouse No. 10. Table 6 shows the distribution route of vehicles corresponding to candidate warehouse No. 3. The specific route and longitude and latitude of route points are shown in Figure 3, and the warehouse No. 10 is equipped with 4 vehicles. The distribution time and time completion efficiency of each demand point are shown in Table 7. The objective function value (total cost) of the distribution scheme is 1023.82, and the time completion efficiency is 1 .

The blue line in the figure represents the convergence trajectory of the squirrel search algorithm, red line represents adaptive particle swarm optimization, yellow line represents the adaptive genetic algorithm, purple line represents chicken swarm optimization, and green line represents the wolf pack algorithm. The results show that its convergence speed and convergence effect are better than other colored lines.

By running these five algorithms 10 times with 1000 iterations each time, in the data volume of $10 \times 13,10 \times 33$, $10 \times 53,10 \times 73$, and $10 \times 93$, as shown in Table 8 , the best solution, average solution, worst solution, and median solution of SSA are better than the other four algorithms, and the solution stability is also higher than other algorithms. And its running time ranks fourth among the five algorithms, less than WPA. Therefore, by comprehensively analyzing the solution results of five different scale examples, SSA is better than the other four algorithms to find the approximate optimal solution of the common storage and common distribution with location and layout model under the multichannel back- ground, with more stable optimization ability and good optimization effect for large data volume examples.

\section{Conclusion}

In this paper, a multichannel common storage and common distribution location model considering time window constraints is established, and the allowable receiving and sending time period of each distribution point is taken as the constraint condition in the design of distribution routes, so as to solve the optimal warehouse location scheme with the minimum total cost and the optimal distribution routes of each channel that conform to the time window constraints. Through the comparison of various intelligent algorithms, it is found that the squirrel search algorithm has better solution effect when solving large data volume examples of the model.

\section{Data Availability}

Data will be made available on request.

\section{Conflicts of Interest}

The author(s) declare(s) that there is no conflict of interest regarding the publication of this paper.

\section{References}

[1] Y. He, X. Wang, Y. Lin, F. Zhou, and L. Zhou, "Sustainable decision making for joint distribution center location choice," Transportation Research Part D: Transport and Environment, vol. 55, no. 8, pp. 202-216, 2017.

[2] L. N. Y. Wong, V. S. K. Lai, and T. P. Y. Tam, "Joint spacing distribution of granites in Hong Kong," Engineering Geology, vol. 245, no. 12, pp. 120-129, 2018.

[3] H. Y. Xu, S. H. Kuo, G. Li, E. F. T. Legara, D. Zhao, and C. P. Monterola, "Generalized cross entropy method for estimating joint distribution from incomplete information," Physica A: Statistical Mechanics and its Applications, vol. 453, no. 6, pp. 162-172, 2016.

[4] W. M. Kempa and R. Marjasz, "Distribution of the time to buffer overflow in the M/G/1/N-type queueing model with batch arrivals and multiple vacation policy," Journal of the Operational Research Society, vol. 71, no. 3, pp. 447-455, 2019.

[5] L. Zhou, R. Baldacci, D. Vigo, and X. Wang, "A multi-depot two-echelon vehicle routing problem with delivery options arising in the last mile distribution," European Journal of Operational Research, vol. 265, no. 2, pp. 765-778, 2018.

[6] J. C. Goodson, "A priori policy evaluation and cyclic-orderbased simulated annealing for the multi-compartment vehicle routing problem with stochastic demands," European Journal of Operational Research, vol. 241, no. 2, pp. 361-369, 2015.

[7] R. Spliet, S. Dabia, and T. van Woensel, "The time window assignment vehicle routing problem with time-dependent travel times," Transportation Science, vol. 52, no. 2, pp. 261276, 2017.

[8] L. Wei, Z. Zhang, D. Zhang, and S. C. H. Leung, "A simulated annealing algorithm for the capacitated vehicle routing problem with two-dimensional loading constraints," European 
Journal of Operational Research, vol. 265, no. 3, pp. 843-859, 2018.

[9] J. Rieck, C. Ehrenberg, and J. Zimmermann, "Many-to-many location-routing with inter-hub transport and multi-commodity pickup-and-delivery," European Journal of Operational Research, vol. 236, no. 3, pp. 863-878, 2014.

[10] J. M. Home-Ortiz, M. Pourakbari-Kasmaei, M. Lehtonen, and J. R. Sanches Mantovani, "Optimal location-allocation of storage devices and renewable-based DG in distribution systems," Electric Power Systems Research, vol. 172, no. 6, pp. 11-21, 2019.

[11] F. Ahmadizar, M. Zeynivand, and J. Arkat, "Two-level vehicle routing with cross-docking in a three-echelon supply chain: a genetic algorithm approach," Applied Mathematical Modelling, vol. 39, no. 22, pp. 7065-7081, 2015.

[12] N. Zhang, X. Zhao, T. Liu, M. Lei, C. Wang, and Y. Wang, "Layout planning of highway transportation environment monitoring network: The case of Xinjiang, China," Sustainability, vol. 12, no. 1, p. 290, 2020.

[13] K. Czarnecki, D. Fourer, F. Auger, and M. Rojewski, “A fast time-frequency multi-window analysis using a tuning directional kernel," Signal Processing, vol. 147, no. 5, pp. 110-119, 2018.

[14] L. W. Rizkallah, M. F. Ahmed, and N. M. Darwish, "SMT-LH: A new satisfiability modulo theory-based technique for solving vehicle routing problem with time window constraints," The Computer Journal, vol. 63, no. 1, pp. 91-104, 2019.

[15] H. F. Liang, "Prediction and simulation of short-term highspeed traffic flow based on wolf swarm algorithm," Computer Simulation, vol. 37, no. 3, pp. 139-143, 2020. 